\title{
Joint adjustment by CPIII precision trigonometric elevation control network assisted with precision levelling observation
}

\author{
Li Jianzhang ${ }^{1,2,3, *}$ \\ ${ }^{1}$ Faculty of Geomatics, Lanzhou Jiaotong University, Lanzhou 730070, China \\ ${ }^{2}$ National-Local Joint Engineering Research Center of Technologies and Applications for National Geographic State Monitoring \\ ${ }^{3}$ Gansu Provincial Engineering Laboratory for National Geographic State Monitoring, Lanzhou 730070, China
}

\begin{abstract}
Using the precision trigonometric elevation instead of the precision levelling to build a CPIII elevation control network will greatly increase the speed of CPIII control network construction. However, the accuracy of CPIII precision trigonometric elevation control network is still difficult to reach the level of CPIII precision levelling network. Based on the existing parameter method, this paper introduces some precision levelling for joint adjustment, and uses Helmert's variance estimation method to perform strict weight determination. Our experiments show that when the number of precision levelling participating in the joint adjustment exceeds $1 / 3$ of the total number of CPIII precision levelling network observations, the accuracy of the CPIII precision trigonometric elevation control network can be effectively improved.
\end{abstract}

\section{Introduction}

A CPIII control network includes a plane control network and an elevation control network, which is the position reference for the construction and operation stages of high-speed railways. The CPIII elevation control network uses electronic level to collect field data. Because the level measurement mode is inefficient, it is a challenges as to how to improve the efficiency of CPIII elevation control network data collection in the current construction of high-speed railway control network.

The CPIII plane control network and the elevation control network have points in common, so some scholars have tried to use the vertical angle and distance in the observation data of the CPIII plane control network to build a CPIII precision trigonometric elevation control network. However, the trigonometric elevation observations are easily affected by systematic errors such as atmospheric refraction, earth curvature, and vertical deviation. In this case, how to eliminate these systematic errors is a key problem to be solved. References [1-5] adopted the observing method to eliminate the influence of atmospheric refraction and the curvature of the earth in the trigonometric elevation observations, so that the precision of the precise trigonometric elevation measurement reached the national second-class requirements. The amount of CPIII control points is huge, and only the measurement prism can be placed near the observation target. The opposite observation method cannot be used to eliminate the effects of atmospheric refraction and earth curvature. Therefore, some researchers have proposed the difference method [6-7] and the parameter method [8], which have achieved good effect. However, from the calculation of a large amount of measured data, it is found that the probability of gross errors in precision trigonometric elevation observations is much higher than the precision levelling. These gross error observations have a serious impact on the control network. In some CPIII precision trigonometric elevationcontrol, In the net, excluding all the gross error observations will result in poor mesh strength or network construction failure.

This paper studies the introduction of part of the precision levelling in the CPIII precise trigonometric elevation control network to improve the strength and reliability of the CPIII precision trigonometric elevation control network. Since two types of observations are involved, Helmert's variance component estimation method is used for weight determination. Through experiments, it is proved that introducing a certain number of precision levelling can effectively improve the accuracy of the CPIII precise trigonometric elevation control network; but if the number of precision levelling is insufficient, it will lead to the failure of strict weight determination.

\section{Joint adjustment model}

As shown in Figure 1, the hollow circles are the CPIII points, the solid circle is the precise trigonometric elevation survey site; the solid lines are the precise trigonometric elevation observation routes, and the dotted lines are the precision levelling observation routes.

\footnotetext{
*Corresponding author: E-mail: 13919054839@163.com
} 


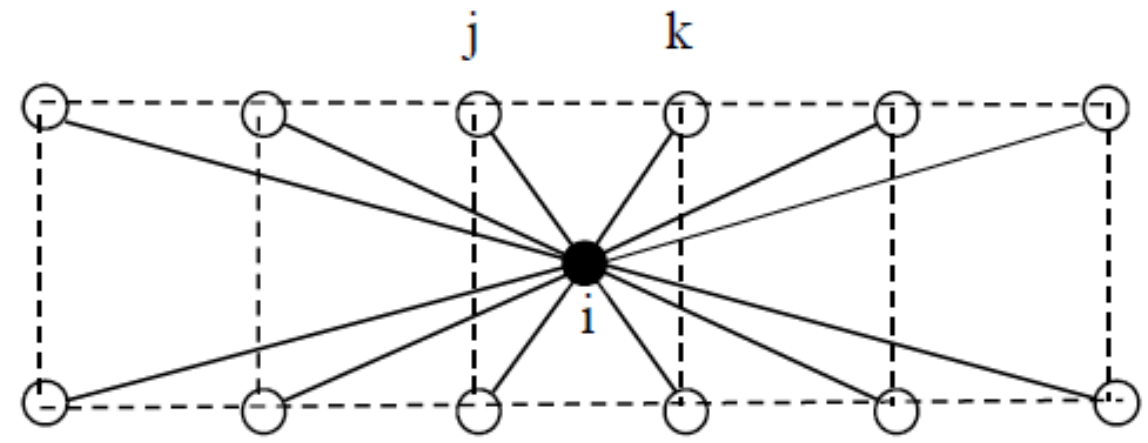

Figure 1: Schematic of Mixed Trigonometric elevation and Precision levelling Observation

Trigonometric elevation measurement is performed on the target point $j$ at the measurement site $i$. Set the vertical angle to $\beta_{i j}$ and the oblique observation value to $s_{i j}$, then we can obtain the trigonometric elevation observation value:

$$
h_{i j}=s_{i j} \sin \beta_{i j}
$$

If the most probable elevation of the instrument center is $y_{i}$ and the most probable elevation of the target point $j$ is $x_{j}$, then:

$$
h_{i j}=x_{j}-y_{i}+k_{i} \times d_{i j}^{2}+\Delta_{i j}
$$

Where $h_{i j}=d_{i j} \times \tan \left(\alpha_{i j}\right)$, and $\Delta_{i j}$ is the true error of the observed value. When the target point $j$ is the point to be obtained, the error equation of the precise trigonometric elevation observation can be obtained as:

$$
v_{i j}=x_{j}-y_{i}+k_{i} \times d_{i j}^{2}-h_{i j}
$$

When the target point $j$ is a known point and the elevation is $H_{j}$, the precise trigonometric elevation observation error equation can be expressed as:

$$
v_{i j}=-y_{i}+k_{i} \times d_{i j}^{2}-\left(h_{i j}-H_{j}\right)
$$

Set the error in the angle measurement to $m_{\alpha}$ and the error in the distance measurement be $m_{s}$. Therefore, the error of the precision trigonometric elevation observation $h_{i j}$ is

$$
m_{i j}^{2}=\left(\sin \left(\alpha_{i j}\right)\right)^{2} m_{s}^{2}+\left(s_{i j} \times \cos \left(\alpha_{i j}\right)\right)^{2} \frac{m_{\alpha}^{2}}{\rho^{2}}
$$

Then its weight $p_{i}=\frac{m_{\alpha}^{2}}{m_{i}^{2}}$.

Combine all the precise trigonometric elevation observation error equations and write the matrix form as:

$$
V_{1}=B_{1} X-l_{1}
$$

Moreover, suppose that level measurement is performed between two points of $j$ and $k$. The observation value is $h_{j k}$, the line length is $s_{j k}$, and the observation error is $\Delta_{j k}$, then:

$$
h_{j k}+\Delta_{j k}=x_{k}-x_{j}
$$

When $j$ and $k$ are both unknown points, the error equation of the precision levelling observation can be expressed as

$$
v_{j k}=x_{k}-x_{j}-h_{j k}
$$

When $j$ is an unknown point, $k$ is a known point, and the elevation is $H_{k}$, the error equation of the precision levelling observation can be obtained as:

$$
\boldsymbol{S}=\left[\begin{array}{c}
n_{1-2 \operatorname{tr}}\left(\mathbf{N}^{-1} \boldsymbol{N}_{1}\right)+{ }_{\operatorname{tr}}\left(\mathbf{N}^{-1} \boldsymbol{N}_{1}\right)^{\wedge} 2 \\
\operatorname{tr}^{\left(\mathbf{N}^{-1} \boldsymbol{N}_{1} \boldsymbol{N}^{-1} \boldsymbol{N}_{2}\right)}
\end{array}\right.
$$$$
\underset{\left.n_{2-2 \operatorname{tr}}\left(\mathbf{N}^{-1} \boldsymbol{N}_{2}\right)+{ }_{\operatorname{tr}}\left(\mathbf{N}^{-1} \boldsymbol{N}_{2}\right)^{\wedge} \boldsymbol{N}^{-1} \boldsymbol{N}_{2}\right)}{W}=\left[\begin{array}{l}
\boldsymbol{V}_{1}{ }^{T} \boldsymbol{P}_{1} \boldsymbol{V}_{1} \\
\boldsymbol{V}_{2}{ }^{T} \boldsymbol{P}_{2} \boldsymbol{V}_{2}
\end{array}\right]
$$

$$
v_{j k}=-x_{j}-\left(h_{j k}-H_{k}\right)
$$
precision levelling observation can be obtained as:

$$
v_{j k}=x_{k}-\left(h_{j k}+H_{j}\right)
$$
equations and write the matrix form as

$$
\boldsymbol{V}_{2}=\boldsymbol{B}_{2} \boldsymbol{X}-\boldsymbol{l}_{2}
$$

\section{The tight adjustment of joint adjustment} value to be $X$, then:

$$
\begin{aligned}
& V_{1}=B_{1} X-l_{1} \\
& V_{2}=B_{2} X-l_{2}
\end{aligned}
$$
method. be obtained

$$
\boldsymbol{\theta}=\boldsymbol{S}^{-1} \boldsymbol{W}
$$

Where:

When the $k$ point is unknown, the $j$ point is a known point, and the elevation is $H_{j}$, the error equation of the

The weight of the precision levelling observation $h_{j k}$ is $p_{j k}=\frac{m_{\alpha}^{2}}{m_{j k}^{2}}$, where $m_{j k}=s_{j k} \times m_{h}$, and $m_{h}$ is the roundtrip error of per kilometer level observation, which is $\sqrt{2}$ times of the nominal accuracy of the precision levelling . Combine all the precision levelling observation error

Combine Eqs.(5) and Eqs.(10), solve the equation using the least square method, and set the estimated parameter

In theory, as the precise trigonometric elevation observations and precision levelling are independent of each other, the addition of precision levelling will not affect the weight of the original CPIII precision trigonometric elevation control network observations. However, the weight ratio relationship between precision levelling and precise trigonometric elevation observations needs to be determined by Helmert variance estimation

Suppose the unit weight variances of the precise trigonometric elevation observations and precision levelling are $\delta_{1}^{2}, \delta_{2}^{2}$ respectively, and let $\boldsymbol{\theta}=\left[\begin{array}{ll}\delta_{1}^{2} & \delta_{1}^{2}\end{array}\right]^{T}$, Based on the Helmert variance estimation method, it can 
$\boldsymbol{N}_{1}=\boldsymbol{B}_{1}^{T} \boldsymbol{P}_{1} \boldsymbol{B}_{1}, \boldsymbol{N}_{2}=\boldsymbol{B}_{2}^{T} \boldsymbol{P}_{2} \boldsymbol{B}_{2}, \boldsymbol{N}=\boldsymbol{B}^{T} \boldsymbol{P} \boldsymbol{B}, n_{1}$ and $n_{2}$ are the number of precise trigonometric elevation observations and precision levelling.

By weighting again, we can get

$$
\boldsymbol{P}_{1}=\frac{\delta_{1}^{2}}{\delta_{1}^{2} \boldsymbol{P}_{1}^{-1}}, \quad \boldsymbol{P}_{2}=\frac{\delta_{1}^{2}}{\delta_{2}^{2} \boldsymbol{P}_{2}^{-1}}
$$

Parameter estimation is performed again using the newly determined weight matrix. Repeat the above process until $\delta_{1}^{2}=\delta_{2}^{2}$ or its ratio is 1 to stop.

\section{Experimental verification}

A CPIII control network with a total length of $10 \mathrm{~km}$ was observed using measuring robots and the electronic precision levelling. Among them, 103 stations of precision trigonometric height measurement obtained 1261 observations (with a few encryption points); a total of 604 precision levelling were obtained. Taking this case as an example, the calculation accuracy of CPIII precision levelling network, CPIII precision trigonometric elevation control network, precision levelling constrained CPIII precision trigonometric elevation control network, precision levelling and precision levelling combined CPIII elevation control network are compared and analyzed.

As shown in Figure 2, after the CPIII precision levelling network is adjusted, the error in the height difference between adjacent points can reach $0.12 \mathrm{~mm}$. The longitudinal observations on both sides of the CPIII precision levelling network are selected to participate in the CPIII precision trigonometric elevation control network. The results are shown in Figure 3. The error in the height difference between adjacent points can reach the level of $0.25 \mathrm{~mm}$. The lateral observations of the line in the CPIII precision levelling network are selected. Participated in the CPIII precision trigonometric elevation control network. The results are shown in Figure 4. The error in the height difference between horizontally adjacent points reached a level of $0.15 \mathrm{~mm}$, and the accuracy of the height difference between adjacent vertical points was small, but only three pairs of height differences exceeded $0.5 \mathrm{~mm}$.

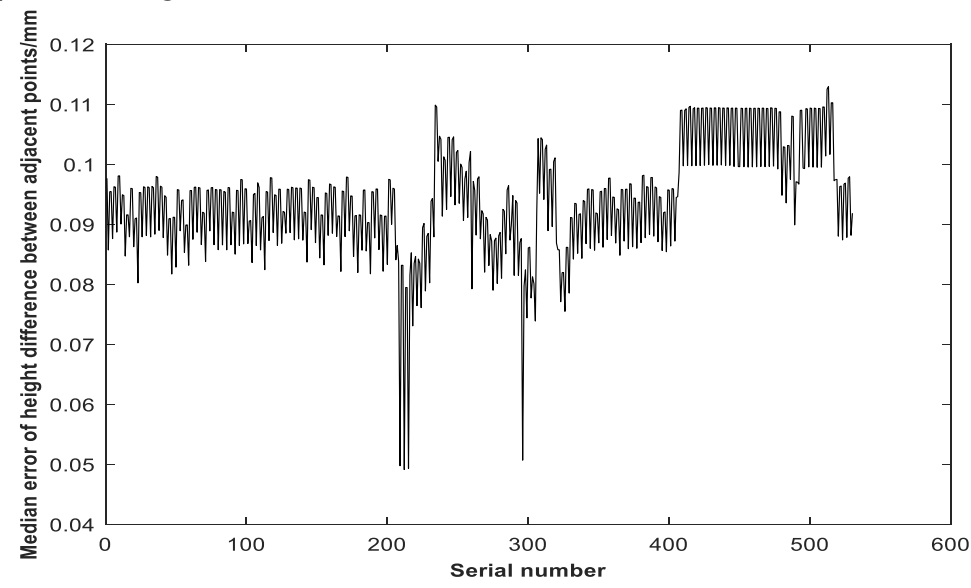

Figure 2: Error curve of height difference between adjacent points of CPIII precision levelling network

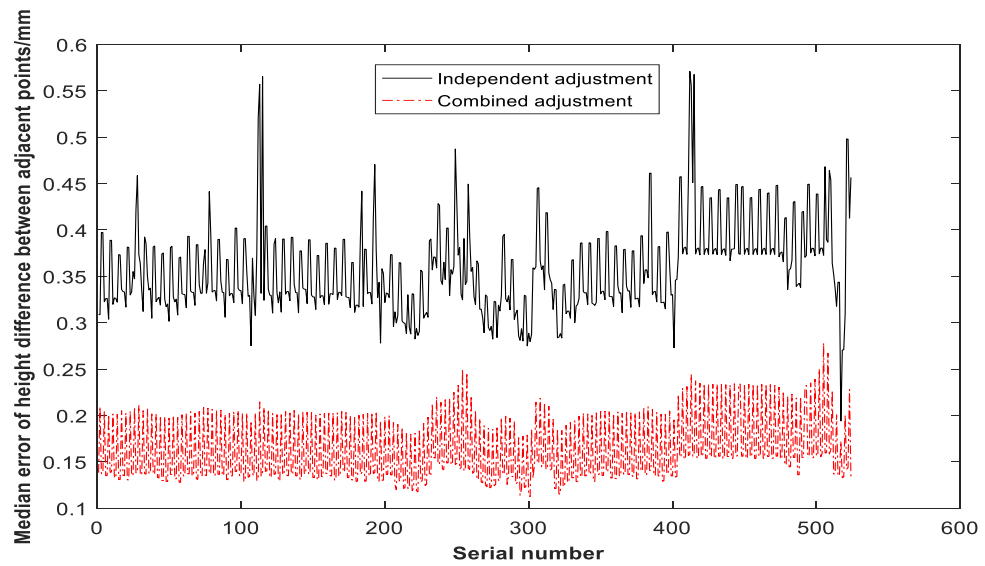

Figure 3: The middle error curve obtained from the joint adjustment of the longitudinal precision levelling and the CPIII precise trigonometric elevation network 


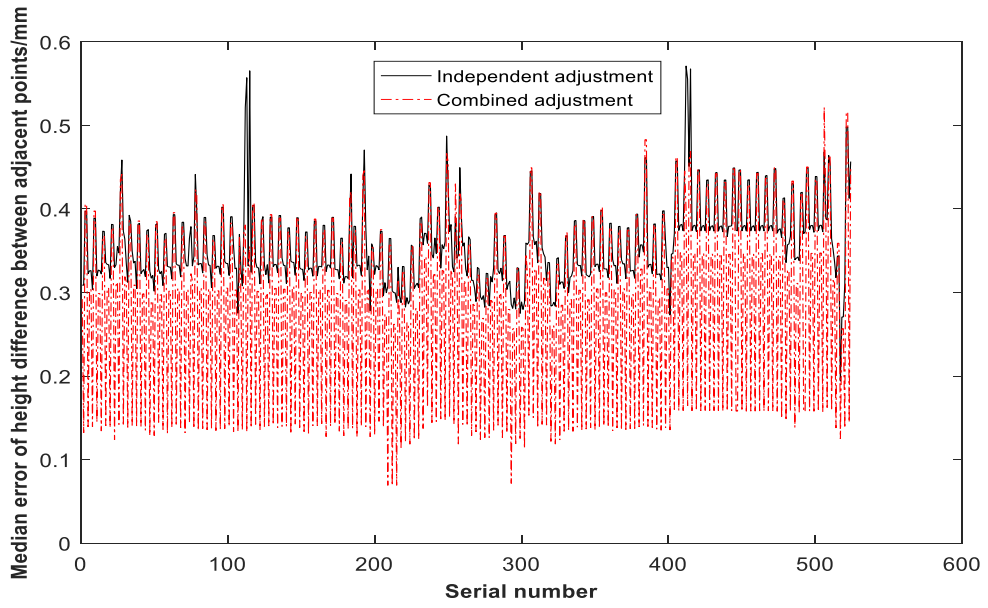

Figure 4: The median error curve obtained from the joint adjustment of the horizontal precision levelling and the CPIII precise trigonometric elevation network

\section{Conclusion}

This paper studies introducing part of precision levelling in the CPIII precision trigonometric elevation control network for joint adjustment to improve its strength and reliability. It can be concluded through experiments that:

1) Normally, adding the longitudinal precision levelling on both sides of the line can effectively improve the accuracy of the CPIII precise trigonometric elevation control network; if only the lateral precision levelling is introduced, the accuracy of the error in the height difference between the lateral CPIII points can be significantly improved. The improvement of error accuracy in the height difference between longitudinal CPIII points is limited.

2) In the case where the number of precision levelling is small, that is, less than $1 / 3$ of the number of CPIII precision levelling network observations, the effect of estimating weights by using the Helmer test backdifference is not good. It is appropriated to adopt nominal accuracy for fixed weight in this case.

\section{Acknowledgments}

This paper can be successfully completed, thanks to my mentor Professor Yan Haowen's strong guidance, here to express sincere respect. At the same time, I would like to express my heartfelt thanks to Gansu Comprehensive Engineering Investigation Institute Co., Ltd. All the experiments in this paper have been helped by the company.

Thank you to my family and my colleagues, and thank you for your selfless dedication, so that I can successfully complete the writing of this article.

Foundation support: The National Natural Science Foundation of China (No. 41661091)

\section{Reference}

1. ZOU Jingui, ZHU Yongchao, XU Yaming. Design and implement of onboard precise trigonometric leveling system based on intelligent total station[J]. Bulletin of Surveying and Mapping, 2014(3):1-5.

2. KONG Ning, LIN Hong, OU Haiping, LIU Yeguang, LUO Feng. Research and application of precise trigonometric leveling to replace second-order leveling based on intelligent total station[J]. Bulletin of Surveying and Mapping, 2016(2):107-109.

3. XU Yaming, SHI Bin, WANG Daixiong, PAN Zhengfeng. An improved trigonometric leveling method for cross-sea elevation transfer[J]. Bulletin of Surveying and Mapping, 2014(4):65-78.

4. WU Dinjun, LI Jiankun, HE Guangyuan. Study of extra-long distance sea-crossing elevation transference based on trigonometric leveling[J]. Science of Surveying and Mapping, 2017, 42(12): 195-200.

5. GUO Zizhen, CHANG Jianzen, YU Chenglei. Exploration and implementation of long distance and high accuracy cross sea quasi measurement under unstable conditions[J]. Bulletin of Surveying and Mapping, 2016(4): 138-139.

6. LIU Chenglong, YANG Xuefeng,LU Jiankang,HE Bo. Construction and adjustment method for CPIII trigonometric leveling network of high-speed railway[J]. Journal of Southwest Jiao Tong University, 2011, 46(3):433-439.

7. FU Jianbin, LIU Chenglong, LU Jiankang, HE Bo, YANG Xuefeng. Study on the CPIII vertical control network establishing method of high speed railway basedon free-station[J]. Journal of Railway Engineering Society, 2010(11): 32-37.

8. LI Jianzhang, YAN Haowen. Parameter method data processing of CPIII Precise Trigonometric vertical control network [J]. Journal of Surveying and mapping, 2019, 48(4): 431-438. 